\title{
Coronary artery bypass grafting and lung lobectomy: Functional outcomes at discharge
}

\author{
Kazuaki Kuwabara ${ }^{1 \star}$, Shinya Matsuda ${ }^{2}$, Kiyohide Fushimi ${ }^{3}$, Koichi B. Ishikawa ${ }^{4}$, Hiromasa \\ Horiguchi $^{5}$ and Kenji Fujimori ${ }^{6}$
}

\footnotetext{
${ }^{1}$ Kyushu University, Graduate School of Medical Sciences, Department of Health Care Administration and Management, Fukuoka, Japan.

${ }^{2}$ Department of Preventive Medicine and Community Health, University of Occupational and Environmental Health, Kitakyushu, Japan.

${ }^{3}$ Department of Health Policy and Informatics, Tokyo Medical and Dental University, Graduate School of Medicine, Tokyo, Japan.

${ }^{4}$ Economics Section, Surveillance Division, Center for Cancer Control and Information Services, National Cancer Center, Tokyo, Japan.

${ }^{5}$ Department of Health Management and Policy, Graduate School of Medicine, The University of Tokyo, Tokyo, Japan.

${ }^{6}$ Center for Regional Healthcare and Certified Educator Support, Hokkaido University, Sapporo, Japan.
}

Accepted 22 February, 2013

\begin{abstract}
Mortality and complication rates after on-pump versus off-pump coronary artery bypass grafting (CABG), and open lung lobectomy (OL) versus video-assisted lobectomy (VAL), have previously been reported, but further research regarding functional outcomes is needed. This study compared functional recovery at discharge between patients who underwent on-pump versus off-pump CABG, and OL versus VAL. In patients discharged during July to December, from 2006 to 2010, functional recovery was measured by comparing the Barthel index at admission and discharge. Complication rates and functional deterioration were compared in propensity score-matched groups of patients aged over 15 years who underwent isolated CABG of two or more arteries or lobectomy, and survived until discharge. The study included 3,901 on-pump CABG patients, 3,672 off-pump CABG patients, 6,029 OL patients, and 14,378 VAL patients. Patient and hospital characteristics, comorbidities, and preoperative care procedures were associated with on-pump versus off-pump CABG, and OL versus VAL, but functional deterioration was not. The complication rate was lower after VAL than OL. Dependent functional status at admission was associated with functional deterioration in patients who underwent lobectomy. Multidisciplinary treatment strategies to maintain functional status should be developed, and appropriate indications for lobectomy according to functional status at admission should be determined.
\end{abstract}

Key words: Coronary artery bypass grafting, complications, functional outcomes, lung lobectomy.

\section{INTRODUCTION}

With the increasing size of the elderly population, the number of disabled elderly individuals is increasing. According to the Organization for Economic Co-operation

*Corresponding author. E-mail: kazu228@basil.ocn.ne.jp. Tel: +81-92-642-6956. Fax: +81-92-642-6961. and Development (OECD) StatExtracts 2011, the proportion of the Japanese population aged over 65 years increased from $9.1 \%$ in 1980 to $23.1 \%$ in 2010 (for the United States, this proportion increased from 11.3 to $13.0 \%$ during the same time period) (OECD StatExtracts, 2011). As a growing number of elderly individuals develop cardiovascular and lung diseases, there is an increasing need for treatments that are less invasive and provide 
better functional outcomes. Previous studies have compared postoperative complication and mortality rates between patients who underwent on-pump versus offpump coronary artery bypass grafting (CABG), and open lung lobectomy (OL) versus video-assisted lobectomy (VAL) (Angelini et al., 2009; Gopaldas et al., 2010; Kapetanakis et al., 2008; Kiernan et al., 2011; Kuss et al., 2010; Lim et al., 2006; Mishra et al., 2006; Motallebzadeh et al., 2006; Rivera et al., 2011).

Although the usefulness of off-pump CABG and VAL have been validated in randomized studies of selected subjects, it is desirable to conduct community-based studies to confirm the safety and effectiveness of minimally invasive surgery (MIS) procedures (off-pump CABG and VAL) versus conventional procedures (on-pump $\mathrm{CABG}$ and $\mathrm{OL}$ ), to assist in determining appropriate procedure choices, and in implementing relevant healthcare policies (Kiernan et al., 2011; Kuss et al., 2010; Lim et al., 2006; Mishra et al., 2006; Rivera et al., 2011).

Previous studies used variable designs and definitions of disease severity (Kuss et al., 2010; Lim et al., 2006). The effects of selection bias have also been discussed (Gopaldas et al., 2010; Kiernan et al., 2011; Mishra et al., 2006; Rivera et al., 2011). Outcomes have been investigated in terms of mortality and complication rates, but functional recovery at discharge has not been sufficiently investigated (Angelini et al., 2009; Kapetanakis et al., 2008; Motallebzadeh et al., 2006). Our Japanese database which includes both functional status information and procedure-based severity information, enables comparisons of functional recovery between propensity score-matched groups of patients who underwent MIS versus conventional procedures. Analysis of such a large population-based database can help to determine the appropriate indications for surgical procedures.

This study used the Japanese administrative database, including data describing functional status at admission and discharge, and the dates and quantities of medical care items used to compare functional outcomes between on-pump versus off-pump CABG and OL versus VAL.

\section{MATERIALS AND METHODS}

\section{Database}

This was a secondary data analysis embedded in a government research project to develop the Japanese case-mix classification. In cooperation with the Ministry of Health, Labour and Welfare (MHLW), our research team started this project in 2001, using the administrative database for 2001 and 2002 to profile hospital performance and develop the payment system. All 82 academic hospitals in Japan participated in this project, and the number of participating community hospitals increased from 92 in 2003 to 1,650 in 2010 (Ministry of Health, Labour and Welfare, 2011). The database includes clinical data as well as claims data such as the date, charge, and quantity of medical care items used. The data of patients discharged between July 1 and October 31, during 2002 through 2005, and between July 1 and December 31 during 2006 through 2009, were collected and merged into a standardized electronic format by the MHLW. Original database included $13,604,026$ patients from the 1,101 acute care hospitals participating in our project from 2004 to 2010. The study protocol was approved by the Ethics Committee of the University of Occupational and Environmental Health, Fukuoka.

\section{Study patients}

Of the $12,502,528$ patients discharged during the 5 years from 2006, 26,472 patients who underwent coronary artery surgery (CABG or intraoperative angioplasty) in 408 hospitals, and 84,564 patients who underwent lung resection (wedge resection, partial lobectomy, lobectomy, or sleeve resection) in 900 hospitals, were identified. Patients who underwent CABG of two or more coronary vessels $(22,506$ patients in 404 hospitals), or resection of one or more lobes with or without sleeve resection $(54,462$ patients in 774 hospitals), were included in this study. Next, we enrolled the patients who were discharged from the hospitals participating in our project during the 5 consecutive years, from 2006 in this study. Patients aged less than 15 years, who had missing barthel index (BI) data, and who underwent aneurysmectomy or valve surgery during the same hospitalization were excluded.

\section{Definitions of variables}

The following variables were compared between on-pump and offpump CABG, and between OL and VAL: age, sex, ambulance use, functional status measured by the $\mathrm{BI}$, weighted comorbidity score measured by the Charlson comorbidity index $(\mathrm{CCl})$, complication rates, length of intensive care unit (ICU) stay, percutaneous coronary intervention (PCI), critical care procedures, blood transfusion, chemoradiation therapy, operating room (OR) time, hospital patient volume, hospital teaching status (academic or community) and fiscal year (FY).

The critical care procedures recorded were pre- and postoperative mechanical ventilation, blood purification (hemodialysis, hemodiafiltration, or hemadsorption), and use of cardiac support devices (intra-aortic balloon pump, percutaneous cardiopulmonary support, or ventricular assist system). Preoperative critical care procedures and blood transfusion were used as markers of preoperative organ failure and anemia, respectively. $\mathrm{PCI}$ included pre- and postoperative thrombolysis, balloon angioplasty, stent insertion, and atherectomy because hybrid coronary artery revascularization was advocated (Holzhey et al., 2008). OR time included the time to implement monitoring, induce anesthesia, position the patient, and perform surgery.

Functional change was measured by the change in $\mathrm{BI}$ during hospitalization in patients who survived to discharge. The BI measures the ability performance in 10 activities of daily living (feeding, grooming, bathing, dressing, bowel and bladder care, toilet use, ambulation, transfers, and stair climbing) on a five-point scale, with scores ranging from 0 (totally dependent) to 100 (fully independent) (Sulter et al., 1999). The BI at admission was categorized as dependent $(<59)$, partially independent requiring assistance (60 to 84 ), or nearly completely independent $(\geq 85)$, because it was expected that change in functional status during hospitalization would be associated with the functional status at admission (Kugler et al., 2003). Functional outcome was defined as $\mathrm{BI}$ at discharge minus $\mathrm{BI}$ at admission, and was categorized as improvement, no change, or deterioration (Kugler et al., 2003).

Age was divided into three categories: 15 to 64,65 to 74 , and $\geq$ 75 years. Up to four comorbidities and four complications were recorded per patient, and were indexed according to the International Classification of Diseases (ICD), 10th edition. The severity of chronic comorbid conditions was assessed using the 
CCl (Sundararajan et al., 2004). Minor and major complications were examined. Minor complications were classified as present if the ICD codes corresponded with wound complications, hematoma and laceration, or disruption of organs by instrumentation or manipulation (T81 to T87) (Zhan and Miller, 2003). Major complications were defined as complications requiring reoperation for hemostasis and evacuation of hematoma, or intra-thoracic abscess. As information regarding surgeon experience was not recorded, hospital CABG and lung resection volumes were averaged over 5 years and divided into three groups: high-volume hospitals (HVHs), medium-volume hospitals (MVHs), and lowvolume hospitals (LVHs); so that the three groups consisted of relatively equal numbers of patients (CABG: 105 LVHs, $\leq 32 / y e a r$; $40 \mathrm{MVHs}, 33$ to 56/year; $25 \mathrm{HVHs}, \geq 57 /$ year. Lung lobectomy: 197 LVHs, $\leq 61$ /year; 50 MVHs, 62 to 103/year; 31 HVHs, $\geq 104 /$ year). Since 2006, hospital fee calculations have depended on the functional status of patients according to the $\mathrm{BI}$ which was recorded every day by well-trained nurses and audited by the MHLW.

\section{Statistical analysis}

Categorical data were compared between the on- and off-pump CABG groups and between the OL and VAL groups using the Chisquare test. Continuous variables were compared between MIS and conventional procedures using analysis of variance. Logistic regression models were used to identify recorded preoperative variables associated with the choice of on-pump versus off-pump CABG, and OL versus VAL. Propensity score-matched cohorts with equal proportions of patients likely to receive on-pump versus offpump CABG, or OL versus VAL, were selected. Postoperative critical care procedures, blood transfusion, complication rates, and deterioration in $\mathrm{BI}$ were compared between these cohorts. Logistic regression models were also used to analyze associations between MIS versus conventional procedures, and complication rates or deterioration in $\mathrm{Bl}$. The model for analysis of complication rates included preoperative critical care procedures, deterioration in $\mathrm{BI}$, and overall critical care procedures. Statistical analyses were performed using International business machines- Statistical package for the social sciences (IBM-SPSS) version 19.0. A value of $p<0.05$ was considered to be statistically significant.

\section{RESULTS}

Of all the patients reviewed in 263 hospitals, 3,901 onpump CABG patients in 164 hospitals (692 in 36 academic hospitals), 3,672 off-pump CABG patients in 152 hospitals (1,126 in 34 academic hospitals), 6,029 OL patients in 225 hospitals $(1,759$ in 36 academic hospitals), and 14,378 VAL patients in 242 hospitals (4,975 in 37 academic hospitals) were identified. Comparisons of patient characteristics between groups showed that the categories of age, sex, and $\mathrm{BI}$ at admission were significantly different between patients who underwent on-pump versus off-pump CABG, and the categories of sex and $\mathrm{CCl}$ were significantly different between patients who underwent OL versus VAL. Comparisons of hospital characteristics between groups showed that there were significant differences in the categories of hospital patient volume and hospital teaching status between patients who underwent on-pump versus off-pump CABG, and OL versus VAL (Table 1).

Preoperative critical care, ICU care, blood transfusion, and mechanical ventilation were more frequent in patients who underwent on-pump versus off-pump CABG. ICU care, blood transfusion, mechanical ventilation, and chemoradiation therapy were more frequent in patients who underwent OL versus VAL. OR time was significantly longer in patients who underwent on-pump versus offpump CABG, and OL versus VAL. The proportions of patients who developed deterioration in $\mathrm{BI}$ were higher in patients who underwent on-pump versus off-pump CABG, and OL versus VAL. The complication rate was higher in patients who underwent OL versus VAL (Table 2).

Age, sex, $\mathrm{CCl}$, preoperative $\mathrm{PCl}$, preoperative blood transfusion, hospital teaching status, hospital patient volume, and $\mathrm{FY}$ were associated with the choice of onpump versus off-pump CABG; and sex, $\mathrm{CCl}, \mathrm{BI}$ at admission, preoperative blood transfusion, preoperative blood purification, hospital teaching status, hospital patient volume, and FY were associated with the choice of OL versus VAL (Table 3 ).

Table 4 shows the intensive care procedures and outcomes in propensity score-matched CABG patients ( $\mathrm{n}$ $=3,672)$ and lobectomy patients $(n=12,002)$. The rates of overall ICU stay, blood transfusion, and mechanical ventilation were higher in patients who underwent onpump versus off-pump CABG, and VAL versus OL. OR time was significantly longer in patients who underwent on-pump versus off-pump CABG, and OL versus VAL. The complication rate was higher in patients who underwent $\mathrm{OL}$ versus VAL, but not in those who underwent on-pump versus off-pump CABG.

Complication rates were associated with the type of lung lobectomy but not the type of CABG (off-pump CABG: odds ratio 1.143 [95\% confidence interval 0.973 to 1.344]; VAL: odds ratio 0.774 [ $95 \%$ confidence interval 0.688 to 0.870$]$ ). Off-pump CABG and VAL were not associated with deterioration in $\mathrm{BI}$. In patients who underwent lobectomy, functional status at admission, mechanical ventilation, and blood transfusion were significantly associated with deterioration in $\mathrm{BI}$, but complications were not (Table 5).

\section{DISCUSSION}

This study evaluated whether undergoing off-pump versus on-pump CABG, or VAL versus OL was associated with complication rates or functional outcomes. After adjustment for patient characteristics, preoperative critical care procedures and hospital characteristics, VAL was associated with a lower complication rate than OL. Complication rates and functional deterioration were not associated with on-pump versus off-pump CABG.

Previous studies evaluated the usefulness of off-pump CABG and VAL based on complication and mortality rates. Quality improvement initiatives in ICUs and multidisciplinary cardiac rehabilitation programs have decreased mortality and complication rates and increased quality of life (Brahmbhatt et al., 2010; Suaya et al., 2009). 
Table 1. Patient characteristics among patients receiving study surgical procedures (\%) [SD].

\begin{tabular}{|c|c|c|c|c|c|c|}
\hline \multirow{2}{*}{ Parameter } & \multicolumn{3}{|c|}{ CABG } & \multicolumn{2}{|c|}{ Lobectomy } & \multirow{2}{*}{$\mathbf{P}$} \\
\hline & On-pump & Off-pump & $\mathbf{P}$ & Open & VAL & \\
\hline Number & 3901 & 3672 & & 6029 & 14378 & \\
\hline $\begin{array}{l}\text { Patient, hospital (academic, } \\
\text { community) }\end{array}$ & 128,36 & 118,34 & & 189,36 & 205,37 & \\
\hline \multicolumn{7}{|l|}{ Age (Years) } \\
\hline $65-74$ & 1568 (40.2) & 1451 (39.5) & & 2319 (38.5) & 5342 (37.2) & 0.147 \\
\hline$\geq 75$ & 1012 (25.9) & $1132(30.8)$ & $<0.001$ & $1573(26.1)$ & 3906 (27.2) & \\
\hline Mean, [SD] & $67.7(9.6)$ & $68.9(9.3)$ & & $67.0(10.8)$ & $67.3(10.5)$ & 0.058 \\
\hline \multicolumn{7}{|l|}{ Gender } \\
\hline Male & $2904(74.4)$ & $2887(78.6)$ & $<0.001$ & $4186(69.4)$ & $8483(59.0)$ & $<0.001$ \\
\hline \multicolumn{7}{|l|}{ Ambulance } \\
\hline Use & $526(13.5)$ & $425(11.6)$ & 0.012 & $22(0.4)$ & $32(0.2)$ & 0.071 \\
\hline \multicolumn{7}{|l|}{ Charlson comorbidity index } \\
\hline 1 & 1415 (36.3) & 1317 (35.9) & & $1386(23.0)$ & 2753 (19.1) & \\
\hline 2 & 834 (21.4) & 779 (21.2) & 00001 & $871(14.4)$ & $2393(16.6)$ & -0001 \\
\hline 3 & 295 (7.6) & 333 (9.1) & $<0.001$ & $422(7.0)$ & $1011(7.0)$ & $<0.001$ \\
\hline 4 or more & $128(3.3)$ & $142(3.9)$ & & $215(3.6)$ & $572(4.0)$ & \\
\hline \multicolumn{7}{|l|}{$\mathrm{BI}$ at admission category } \\
\hline Dependent & $521(13.4)$ & $388(10.6)$ & 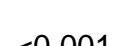 & $47(0.8)$ & $133(0.9)$ & 0182 \\
\hline Partially independent & $128(3.3)$ & $117(3.2)$ & $<0.001$ & $70(1.2)$ & $133(0.9)$ & 0.183 \\
\hline \multicolumn{7}{|l|}{ Hospital patient volume } \\
\hline LVH & $1396(56.2)$ & $1088(43.8)$ & & $1942(32.8)$ & $3978(67.2)$ & \\
\hline MVH & $1007(44.1)$ & $1275(55.9)$ & $<0.001$ & $1938(28.0)$ & $4978(72.0)$ & $<0.001$ \\
\hline $\mathrm{HVH}$ & $1498(53.4)$ & $1309(46.6)$ & & $2149(28.4)$ & $5422(71.6)$ & \\
\hline \multicolumn{7}{|l|}{ Teaching status } \\
\hline Academic & $692(17.7)$ & $1126(30.7)$ & $<0.001$ & 1759 (29.2) & 4975 (34.6) & $<0.001$ \\
\hline \multicolumn{7}{|l|}{ Fiscal year } \\
\hline 2006 & $980(50.9)$ & 945 (49.1) & & 1475 (34.8) & $2758(65.2)$ & \\
\hline 2007 & $601(47.7)$ & 659 (52.3) & & 1027 (35.5) & $1862(64.5)$ & \\
\hline 2008 & $643(54.4)$ & $540(45.6)$ & 0.007 & $1022(34.0)$ & $1982(66.0)$ & $<0.001$ \\
\hline 2009 & $692(51.0)$ & $665(49.0)$ & & $1194(29.9)$ & $2802(70.1)$ & \\
\hline 2010 & 985 (53.3) & 863 (46.7) & & $1311(20.9)$ & 4974 (79.1) & \\
\hline
\end{tabular}

SD: Standard deviation. $\dagger$ : compared using analysis of variance. Other comparisons were made using chi-square test. CABG: coronary artery bypass graft, VAL: video assisted lobectomy, BI: barthel index, HVH: high volume hospital, LVH: low volume hospital, MVH: middle volume hospital.

2009). It is important to conduct studies of functional outcomes after MIS procedures to determine efficient allocation of healthcare financing and to improve outcomes in elderly patients (OECD StatExtracts, 2011; Suzuki, 2009).

In this study, the use of VAL was found to increase over the years, but the use of off-pump CABG was not. Some minimally invasive procedures may not have significant advantages over conventional procedures. For example, it was determined that the differences in outcomes after laparoscopic versus open appendectomy did not justify the increased costs of laparoscopic surgery, and the type of appendectomy performed is now determined by the preferences of patients and physicians (Cothren et al., 2005). 
Table 2. Care process and outcomes among patients receiving study surgical procedures (\%) [SD] (Cont).

\begin{tabular}{|c|c|c|c|c|c|c|}
\hline \multirow{2}{*}{ Parameter } & \multicolumn{3}{|c|}{ CABG } & \multicolumn{3}{|c|}{ Lobectomy } \\
\hline & On-pump & Off-pump & $\mathbf{P}$ & Open & VAL & $\mathbf{P}$ \\
\hline \multicolumn{7}{|l|}{ PCl } \\
\hline Overall & $254(6.5)$ & $237(6.5)$ & 0.920 & \multirow{3}{*}{\multicolumn{3}{|c|}{$\star \star \star *$}} \\
\hline Preoperative & 125 (3.2) & $118(3.2)$ & 0.982 & & & \\
\hline Postoperative & $132(3.4)$ & $125(3.4)$ & 0.961 & & & \\
\hline \multicolumn{7}{|l|}{ ICU stay } \\
\hline Overall & 3375 (86.5) & $2989(81.4)$ & $<0.001$ & 2396 (39.7) & $4080(28.4)$ & $<0.001$ \\
\hline Preoperative & $615(15.8)$ & $422(11.5)$ & $<0.001$ & $16(0.3)$ & $16(0.1)$ & 0.011 \\
\hline Postoperative & $3337(85.5)$ & $2958(80.6)$ & $<0.001$ & $2392(39.7)$ & $4076(28.3)$ & $<0.001$ \\
\hline \multicolumn{7}{|l|}{ Blood transfusion } \\
\hline Overall & $2890(74.1)$ & $2003(54.5)$ & $<0.001$ & $663(11.0)$ & $508(3.5)$ & $<0.001$ \\
\hline Preoperative & $90(2.3)$ & 117 (3.2) & 0.019 & $40(0.7)$ & $35(0.2)$ & $<0.001$ \\
\hline Postoperative & $2885(74.0)$ & $1996(54.4)$ & $<0.001$ & $651(10.8)$ & $487(3.4)$ & $<0.001$ \\
\hline \multicolumn{7}{|l|}{ Ventilation } \\
\hline Overall & $2767(70.9)$ & $2171(59.1)$ & $<0.001$ & $226(3.7)$ & $217(1.5)$ & $<0.001$ \\
\hline Preoperative & $99(2.5)$ & $77(2.1)$ & 0.203 & $6(0.1)$ & $4(0.0)$ & 0.035 \\
\hline Postoperative & $2751(70.5)$ & $2153(58.6)$ & $<0.001$ & $223(3.7)$ & $215(1.5)$ & $<0.001$ \\
\hline \multicolumn{7}{|l|}{ Cardiac support device } \\
\hline Overall & $740(19.0)$ & $637(17.3)$ & 0.067 & \multirow{3}{*}{\multicolumn{3}{|c|}{ *** }} \\
\hline Preoperative & $244(6.3)$ & $172(4.7)$ & 0.003 & & & \\
\hline Postoperative & $681(17.5)$ & $604(16.4)$ & 0.243 & & & \\
\hline \multicolumn{7}{|l|}{ Blood purification } \\
\hline Overall & $394(10.1)$ & $355(9.7)$ & 0.529 & $38(0.6)$ & $123(0.9)$ & 0.097 \\
\hline Preoperative & $273(7.0)$ & $274(7.5)$ & 0.436 & $30(0.5)$ & $106(0.7)$ & 0.055 \\
\hline Postoperative & $386(9.9)$ & $345(9.4)$ & 0.462 & $38(0.6)$ & $120(0.8)$ & 0.129 \\
\hline \multicolumn{7}{|l|}{ Chemoradiation } \\
\hline Chemotherapy & \multirow{2}{*}{\multicolumn{3}{|c|}{ *** }} & $231(3.8)$ & 454 (3.2) & \multirow[t]{3}{*}{$<0.001$} \\
\hline Radiation & & & & $21(0.3)$ & $18(0.1)$ & \\
\hline Chemoradiation & & & & $23(0.4)$ & $16(0.1)$ & \\
\hline Operating room time (min) & $475.5[200.8]$ & $409.1[103.1]$ & $<0.001^{\dagger}$ & $328.4[121.7]$ & $280.5[231]$ & $<0.001^{\dagger}$ \\
\hline \multicolumn{7}{|l|}{ Complications } \\
\hline Minor complication & $798(20.5)$ & $698(19.0)$ & 0.199 & $704(11.7)$ & $1652(11.5)$ & $<0.001$ \\
\hline Major complication & $16(0.4)$ & $11(0.3)$ & & $31(0.5)$ & $21(0.1)$ & \\
\hline Abscess drainage & $3(0.1)$ & $5(0.1)$ & 0.428 & $17(0.3)$ & $7(0.0)$ & $<0.001$ \\
\hline Operative hemostasis & $14(0.4)$ & $6(0.2)$ & 0.098 & $16(0.3)$ & $14(0.1)$ & 0.004 \\
\hline \multicolumn{7}{|l|}{ BI change } \\
\hline Deterioration & $235(6.0)$ & $203(5.5)$ & $<0.001$ & $155(2.6)$ & $299(2.1)$ & 0.006 \\
\hline
\end{tabular}

†: Compared using analysis of variance. Other comparisons were made using chi-square test. SD: standard deviation, BI: barthel index, CABG: coronary artery bypass graft, VAL: video assisted lobectomy, ICU: intensive care unit, PCI: percutaneous coronary intervention, ${ }^{\star \star *}$ : not examined. 
Table 3. Factors associated with off-pump CABG and VAL.

\begin{tabular}{|c|c|c|}
\hline \multirow{2}{*}{ Parameter } & Off pump CABG & VAL \\
\hline & Odds ratio $(95 \% \mathrm{Cl})$ & Odds ratio $(95 \% \mathrm{Cl})$ \\
\hline \multicolumn{3}{|l|}{ Age (for 1564 years) } \\
\hline $65-74$ years & $1.172(1.049-1.309)$ & $0.987(0.919-1.060)$ \\
\hline$\geq 75$ years & $1.468(1.298-1.660)$ & $1.073(0.991-1.161)$ \\
\hline \multicolumn{3}{|l|}{ Gender } \\
\hline Male & 1.335 (1.194-1.493) & $0.643(0.603-0.687)$ \\
\hline \multicolumn{3}{|l|}{ Ambulance } \\
\hline Use & $0.945(0.803-1.113)$ & $0.778(0.435-1.391)$ \\
\hline \multicolumn{3}{|l|}{ Charlson comorbidity index } \\
\hline 1 & $1.077(0.961-1.207)$ & $0.805(0.744-0.871)$ \\
\hline 2 & $1.105(0.967-1.262)$ & $1.100(1.005-1.204)$ \\
\hline 3 & $1.304(1.080-1.575)$ & $0.960(0.848-1.087)$ \\
\hline 4 or more & $1.373(1.045-1.805)$ & $1.055(0.894-1.246)$ \\
\hline \multicolumn{3}{|l|}{$\begin{array}{l}\mathrm{BI} \text { at admission category (for nearly } \\
\text { completely independent) }\end{array}$} \\
\hline Dependent & $0.914(0.772-1.082)$ & $1.293(0.909-1.840)$ \\
\hline Partially independent & $0.870(0.668-1.134)$ & $0.687(0.509-0.929)$ \\
\hline Preoperative PCI & $1.368(1.031-1.814)$ & - \\
\hline Preoperative ICU stay & $0.724(0.606-0.866)$ & $0.608(0.283-1.307)$ \\
\hline Preoperative Blood transfusion & $1.615(1.188-2.196)$ & $0.412(0.255-0.665)$ \\
\hline Preoperative Ventilation & $0.958(0.683-1.344)$ & $0.754(0.177-3.204)$ \\
\hline Preoperative Blood purification & $0.973(0.798-1.186)$ & $1.529(1.006-2.324)$ \\
\hline Preoperative Cardiac support device & $0.884(0.691-1.130)$ & - \\
\hline \multicolumn{3}{|l|}{ Teaching status (for community) } \\
\hline Academic & $2.048(1.829-2.293)$ & $1.180(1.099-1.266)$ \\
\hline \multicolumn{3}{|l|}{ Hospital patient volume } \\
\hline MVH & $1.492(1.326-1.679)$ & $1.241(1.146-1.344)$ \\
\hline $\mathrm{HVH}$ & $0.975(0.870-1.092)$ & $1.198(1.107-1.297)$ \\
\hline \multicolumn{3}{|l|}{ Fiscal year } \\
\hline 2007 & $1.256(1.085-1.453)$ & $0.952(0.861-1.052)$ \\
\hline 2008 & $0.931(0.801-1.081)$ & $1.018(0.921-1.125)$ \\
\hline 2009 & $1.025(0.888-1.182)$ & $1.251(1.139-1.374)$ \\
\hline 2010 & $0.869(0.761-0.992)$ & $2.024(1.852-2.213)$ \\
\hline \multicolumn{3}{|c|}{ Hosmer Lemeshow goodness of model fit } \\
\hline$p$-value & 0.266 & 0.026 \\
\hline
\end{tabular}

CABG: Coronary artery bypass graft, VAL: video assisted lobectomy, $\mathrm{Cl}$ : confidence interval, $\mathrm{BI}$ : barthel index, $\mathrm{PCl}$ : percutaneous coronary intervention, ICU: intensive care unit, HVH: high volume hospital. LVH: low volume hospital. $\mathrm{MVH}$ : middle volume hospital.

Researchers may well discover other advantages of MIS procedures that would justify our study (Angelini et al., 2009; Kapetanakis et al.,2008; Kiernan et al., 2011; Motallebzadeh et al., 2006; Rivera et al., 2011).
Administrative databases such as the one used in this study are useful for the evaluation of functional outcomes after MIS procedures. Studies in stroke patients found that functional status was low immediately after discharge 
Table 4. Care process and outcomes among the propensity score matched patients receiving study surgical procedures (\%) [SD].

\begin{tabular}{|c|c|c|c|c|c|c|}
\hline \multirow{2}{*}{ Parameter } & \multicolumn{3}{|c|}{ CABG } & \multicolumn{3}{|c|}{ Lobectomy } \\
\hline & On pump & Off pump & $\mathbf{P}$ & Open & VAL & $\mathbf{P}$ \\
\hline Number of matched pairs & 1836 & 1836 & & 6001 & 6001 & \\
\hline \multicolumn{7}{|l|}{$\mathrm{PCl}$} \\
\hline Overall & $133(7.2)$ & $115(6.3)$ & 0.237 & & & \\
\hline Preoperative & $67(3.6)$ & $54(2.9)$ & 0.229 & & $* * *$ & \\
\hline Postoperative & $68(3.7)$ & $63(3.4)$ & 0.656 & & & \\
\hline \multicolumn{7}{|l|}{ ICU stay } \\
\hline Overall & $1580(86.1)$ & $1386(75.5)$ & $<0.001$ & $2382(39.5)$ & $1551(10.8)$ & $<0.001$ \\
\hline Preoperative & $322(17.5)$ & 303 (16.5) & 0.404 & $10(0.2)$ & $8(0.1)$ & 0.637 \\
\hline Postoperative & $1561(85.0)$ & $1368(74.5)$ & $<0.001$ & $2380(39.5)$ & $1550(10.8)$ & $<0.001$ \\
\hline \multicolumn{7}{|l|}{ Blood transfusion } \\
\hline Overall & $1342(73.1)$ & $956(52.1)$ & $<0.001$ & $643(10.7)$ & $214(1.5)$ & $<0.001$ \\
\hline Preoperative & $35(1.9)$ & $39(2.1)$ & 0.639 & $24(0.4)$ & $23(0.2)$ & 0.884 \\
\hline Postoperative & $1338(72.9)$ & $953(51.9)$ & $<0.001$ & $636(10.5)$ & $202(1.4)$ & $<0.001$ \\
\hline \multicolumn{7}{|l|}{ Ventilation } \\
\hline Overall & $1275(69.4)$ & 1077 (58.7) & $<0.001$ & 217 (3.6) & $97(0.7)$ & $<0.001$ \\
\hline Preoperative & $53(2.9)$ & $43(2.3)$ & 0.301 & $1(0.0)$ & $2(0.0)$ & 0.564 \\
\hline Postoperative & $1264(68.8)$ & $1068(58.2)$ & $<0.001$ & $216(3.6)$ & $96(0.7)$ & $<0.001$ \\
\hline \multicolumn{7}{|l|}{ Cardiac support device } \\
\hline Overall & $362(19.7)$ & $372(20.3)$ & 0.680 & & & \\
\hline Preoperative & $132(7.2)$ & $115(6.3)$ & 0.263 & & $* * *$ & \\
\hline Postoperative & $330(18.0)$ & $356(19.4)$ & 0.271 & & & \\
\hline \multicolumn{7}{|l|}{ Blood purification } \\
\hline Overall & $178(9.7)$ & $159(8.7)$ & 0.277 & $38(0.6)$ & $41(0.3)$ & 0.735 \\
\hline Preoperative & $127(6.9)$ & $120(6.5)$ & 0.645 & $30(0.5)$ & $32(0.2)$ & 0.799 \\
\hline Postoperative & $173(9.4)$ & $153(8.3)$ & 0.246 & $38(0.6)$ & $41(0.3)$ & 0.735 \\
\hline \multicolumn{7}{|l|}{ Chemoradiation } \\
\hline Chemotherapy & & & & $228(3.8)$ & $233(1.6)$ & 0.002 \\
\hline Radiation & & $* * *$ & & $21(0.3)$ & $4(0.0)$ & \\
\hline Chemoradiation & & & & $23(0.4)$ & $12(0.1)$ & \\
\hline Operating room time (min) & $467.3[256.2]$ & $406.4[98.1]$ & $<0.001$ & $328.4[121.5]$ & $288.8[332.4]$ & $<0.001$ \\
\hline \multicolumn{7}{|l|}{ Complications } \\
\hline Minor complication & $373(20.3)$ & $412(22.4)$ & 0.214 & $702(11.6)$ & $597(4.2)$ & $<0.001$ \\
\hline Major complication & $8(0.4)$ & $5(0.3)$ & & $31(0.5)$ & $7(0.0)$ & \\
\hline Abscess drainage & $3(0.1)$ & $3(0.1)$ & 1.000 & $17(0.3)$ & $1(0.0)$ & $<0.001$ \\
\hline Operative hemostasis & $6(0.3)$ & $2(0.1)$ & 0.157 & $16(0.3)$ & $6(0.0)$ & 0.033 \\
\hline \multicolumn{7}{|l|}{ BI change category } \\
\hline Deterioration & $101(5.5)$ & $92(5.0)$ & 0.207 & $150(2.5)$ & $127(0.9)$ & 0.374 \\
\hline
\end{tabular}

$\dagger$ : Compared using analysis of variance. Other comparisons were made using chi-square test. SD: standard deviation, CABG: coronary artery bypass graft, VAL: video assisted lobectomy, BI: barthel index, PCl: percutaneous coronary intervention. ${ }^{* * *}$ : not examined. 
Table 5. Factors associated with complications and BI deterioration.

\begin{tabular}{|c|c|c|c|c|}
\hline \multirow{3}{*}{ Parameter } & \multicolumn{2}{|c|}{ Complications } & \multicolumn{2}{|c|}{ BI deterioration } \\
\hline & CABG & Lobectomy & CABG & Lobectomy \\
\hline & Odds ratio $(95 \% \mathrm{Cl})$ & Odds ratio $(95 \% \mathrm{Cl})$ & Odds ratio $(95 \% \mathrm{Cl})$ & Odds ratio $(95 \% \mathrm{Cl})$ \\
\hline \multicolumn{5}{|l|}{ Age (for $15-64$ years) } \\
\hline $65-74$ years & $0.889(0.737-1.073)$ & $1.058(0.919-1.217)$ & $1.676(1.090-2.576)$ & $1.863(1.285-2.700)$ \\
\hline$\geq 75$ years & $0.872(0.697-1.091)$ & $1.173(1.010-1.362)$ & $4.505(2.901-6.996)$ & $4.230(2.976-6.014)$ \\
\hline \multicolumn{5}{|l|}{ Gender } \\
\hline Male & $0.920(0.763-1.110)$ & $0.951(0.837-1.079)$ & $0.957(0.683-1.342)$ & $0.951(0.725-1.248)$ \\
\hline \multicolumn{5}{|l|}{ Ambulance } \\
\hline Use & $1.218(0.928-1.598)$ & $0.878(0.254-3.036)$ & $0.982(0.585-1.648)$ & $0.670(0.079-5.694)$ \\
\hline \multicolumn{5}{|c|}{ Charlson comorbidity index } \\
\hline 1 & $1.506(1.228-1.846)$ & $1.248(1.08-1.442)$ & $0.781(0.532-1.147)$ & $1.373(1.015-1.856)$ \\
\hline 2 & 1.665 (1.326-2.092) & $1.264(1.065-1.500)$ & $1.052(0.696-1.59)$ & $1.190(0.820-1.727)$ \\
\hline 3 & $1.225(0.845-1.777)$ & $0.978(0.770-1.242)$ & $0.781(0.380-1.604)$ & $1.656(1.086-2.526)$ \\
\hline 4 or more & $1.543(0.930-2.561)$ & $1.671(1.277-2.188)$ & $1.486(0.720-3.067)$ & $1.562(0.886-2.754)$ \\
\hline \multicolumn{5}{|c|}{$\begin{array}{l}\mathrm{BI} \text { at admission category (for } \\
\text { nearly completely } \\
\text { independent) }\end{array}$} \\
\hline Dependent & $1.006(0.766-1.321)$ & $1.114(0.556-2.233)$ & $0.320(0.175-0.586)$ & $3.511(1.571-7.846)$ \\
\hline Partially independent & $1.178(0.773-1.794)$ & $0.979(0.576-1.665)$ & $1.023(0.521-2.007)$ & $4.543(2.607-7.918)$ \\
\hline $\mathrm{PCl}^{\dagger}$ & $1.183(0.732-1.911)$ & $\star * *$ & $0.639(0.301-1.357)$ & $* * *$ \\
\hline ICU stay $^{\dagger}$ & $1.041(0.783-1.384)$ & $1.559(0.431-5.637)$ & $1.146(0.741-1.774)$ & $1.199(0.924-1.555)$ \\
\hline Blood transfusion $^{\dagger}$ & $0.740(0.371-1.474)$ & $1.028(0.422-2.502)$ & $1.329(0.892-1.981)$ & $1.958(1.368-2.803)$ \\
\hline Ventilation $^{\dagger}$ & $0.690(0.380-1.255)$ & - & $1.256(0.887-1.779)$ & $3.331(2.120-5.234)$ \\
\hline Blood purification $^{\dagger}$ & $0.730(0.503-1.059)$ & $1.870(0.959-3.649)$ & 3.079 (1.973-4.803) & $2.981(1.290-6.888)$ \\
\hline Cardiac support device ${ }^{\dagger}$ & $0.646(0.432-0.967)$ & $* \star *$ & $1.162(0.782-1.725)$ & $* * *$ \\
\hline \multicolumn{5}{|c|}{ Chemotherapy or radiation } \\
\hline Chemotherapy & & $0.868(0.615-1.227)$ & & $1.381(0.771-2.475)$ \\
\hline Radiation & $* * *$ & $0.844(0.191-3.728)$ & $* * *$ & $3.247(0.684-15.419)$ \\
\hline Chemoradiation & & $0.296(0.040-2.212)$ & & ** \\
\hline
\end{tabular}


Table 5. Contd.

\section{Use of innovation \\ Off pump or VAL}

Complication

\section{Teaching status (for \\ community)}

Academic

\section{Hospital patient volume (fo \\ LVH) \\ $\mathrm{MVH}$}

$\mathrm{HVH}$

\section{Fiscal year (for 2006)}

2007

2008

2009

2010

\section{Operating room time}

One more minute longer
$1.143(0.973-1.344)$

$* * *$

$0.984(0.689-1.407)$

$1.016(0.795-1.298)$

$1.288(1.079-1.537)$

$1.256(0.937-1.685)$

$1.955(1.499-2.550)$

$2.238(1.726-2.903)$

$2.201(1.723-2.811)$

$1.0001(0.9998-1.0005)$
$0.774(0.688-0.870)$

***

$0.720(0.623-0.832)$

1.593 (1.365-1.860)

$1.146(0.982-1.338)$

1.265 (0.999-1.602)

1.625 (1.300-2.032)

$2.039(1.655-2.511)$

$5.014(4.152-6.055)$

$1.000(0.9999-1.0003)$
$0.706(0.344-1.447)$

$1.133(0.842-1.523)$

$1.049(0.769-1.432)$

$1.306(0.921-1.851)$

1.303 (0.827-2.052)

$1.388(0.977-1.972)$

$0.731(0.423-1.265)$

$0.532(0.310-0.913)$

$0.776(0.474-1.270)$

$1.328(0.890-1.981)$
$1.168(0.852-1.602)$

$0.701(0.500-0.981)$

$1.341(0.854-2.106)$

$1.380(0.877-2.172)$

$1.721(1.142-2.592)$

2.557 (1.747-3.742)
$0.559(0.365-0.857)$

\section{Hosmer Lemeshow goodness}

of model fit

\section{$P$-value}

0.094

0.311

0.587

0.577

CABG: coronary artery bypass graft. VAL: video assisted lobectomy, $\mathrm{Cl}$ : confidence interval, BI: barthel index, PCI: percutaneous coronary intervention, ICU: intensive care unit, HVH: high volume hospital, MVH: middle volume hospital, LVH: low volume hospital, ${ }^{\star \star \star}$ : originally not included, ${ }^{\star \star}$ : no cases with BI deterioration and chemoradiation, $\dagger$ : preoperative critical care use in the complication model and overall use during the hospitalization in the $\mathrm{BI}$ deterioration model.

and started to recover after about 30 days, reaching a plateau at 90 to 180 days (Dowdy et al., 2005; Sacanella et al., 2011; Sulter et al., 1999). In this study, the mean lengths of stay were 32 or 37 days for CABG and 15 or 22 days for lobectomy, which is longer than the lengths of stay reported in Western countries (Gopaldas et al.,
2010; Kiernan et al., 2011; Mishra et al., 2006; Rivera et al., 2011). The OECD has acknowledged that acute care hospitals have different roles in Japan than in Western countries (OECD, 2005). The longer length of stay in Japanese hospitals gives us additional information regarding postoperative functional outcomes. In this study, functional outcomes were not significantly better after off-pump versus on-pump CABG, which is consistent with the results of other studies that measured quality of life using the Short-Form 36, and found no significant differences in general health status at 30 to 360 days between patients who underwent on-pump versus off-pump CABG 
(Angelini et al., 2009; Kapetanakis et al., 2008; Motallebzadeh et al., 2006). Functional deterioration was not significantly different after VAL versus OL, although the complication rate was lower after VAL. This finding could provide further validation of the usefulness of VAL, in combination with the findings of other studies that evaluated the advantages and disadvantages of VAL versus OL (Kiernan et al., 2011).

As lower $\mathrm{Bl}$ at admission and advancing FY were associated with higher complication rates and worse functional outcomes in patients who underwent lobectomy, policymakers may pay more attention to promoting VAL than off-pump CABG. Patients who underwent lobectomy may have had different preoperative characteristics than those who underwent CABG. Unlike CABG, lobectomy inherently reduces vital organ capacity, and functional recovery is therefore expected to be less after lobectomy than after CABG. As pre- and postoperative, some critical care procedures were associated with $\mathrm{BI}$ deterioration in $\mathrm{BI}$ for lobectomy patients; it is more important to monitor the quality of critical care rather than in CABG patients. As functional status at admission was associated with functional outcomes in this study, as well as in other studies, appropriate indications for lobectomy should be determined, and relevant skills training and coordination of multidisciplinary treatment among intensivists, cardiologists, OR staff, and rehabilitation staff should be encouraged (Brahmbhatt et al., 2010; Holzhey et al., 2008; Suaya, 2009).

This study has some limitations that should be considered. First, only data from patients discharged during a 6 -month period each year for 5 years were analyzed. Even though patients were matched for many significant covariates that could affect the choice of MIS versus conventional procedures, there may be additional variables associated with this choice that were not taken into consideration. The database has now started to record the postal codes of patients, and the study period has been extended to include the whole year from 2010. The methodology of future observational studies can therefore be strengthened by analyzing the distance between home and hospital and by using a larger database (Suaya et al., 2009).

Second, some variables such as the American Society of Anesthesiologists score and cancer stage were not included in the analyses. As there are concerns that these scales do give precise indications of functional status, the current study analyzed intensive care procedures, indicating organ failure and advanced cancer stage instead. For example, use of intra-aortic balloon pumping indicates severe coronary artery disease, and administration of chemoradiation therapy indicates advanced cancer stage. These analyses also support the development of quality improvement initiatives for critical care procedures (Brahmbhatt et al., 2010).

Finally, patients aged less than 15 years or with missing $\mathrm{BI}$ data were excluded from our analyses. Although the differences between the included and excluded patient groups appear to be significant, these differences are not large enough to distort our results (Annex Table 1).

\section{Conclusion}

This study evaluated differences in complication rates and functional outcomes between patients who underwent on-pump versus off-pump CABG, and OL versus VAL. In propensity score-matched groups of patients who underwent CABG and lobectomy, postoperative complication rates were lower in patients who underwent VAL versus $O L$, but not off-pump versus on-pump CABG. Functional deterioration was not significantly different between MIS and conventional procedures for either CABG or lobectomy. As the physical status at admission was associated with functional outcome in patients who underwent lobectomy, the appropriate indications for VAL should be determined, and perioperative treatment strategies to maintain functional status during hospitalization should be implemented.

\section{ACKNOWLEDGEMENT}

This study was funded in part by Grants-in-Aid for Research on Policy Planning and Evaluation from the Ministry of Health, Labour and Welfare, Japan (H19 seisaku-sitei 001) and from Ministry of Education, Culture, Sports, Science and Technology, Japan (H22 Kiban-Kenkyu C).

\section{REFERENCES}

Angelini GD, Culliford L, Smith DK, Hamilton MC, Murphy GJ, Ascione R, Baumbach A, Reeves BC (2009). Effects of on- and off-pump coronary artery surgery on graft patency, survival, and health-related quality of life: long-term follow-up of 2 randomized controlled trials. J. Thorac. Cardiovasc. Surg. 137(2):295-303.

Brahmbhatt N, Murugan R, Milbrandt EB (2010). Early mobilization improves functional outcomes in critically ill patients. Crit. Care 14(5):321.

Cothren CC, Moore EE, Johnson JL, Moore JB, Ciesla DJ, Burch JM (2005). Can we afford to do laparoscopic appendectomy in an academic hospital? Am. J. Surg. 190(6):950-4.

Dowdy DW, Eid MP, Sedrakyan A, Mendez-Tellez PA, Pronovost PJ, Herridge MS, Needham DM (2005). Quality of life in adult survivors of critical illness: a systematic review of the literature. Intensive Care Med. 31(5):611-620.

Gopaldas RR, Bakaeen FG, Dao TK, Walsh GL, Swisher SG, Chu D (2010). Video-assisted thoracoscopic versus open thoracotomy lobectomy in a cohort of 13,619 patients. Ann. Thorac. Surg. 89(5):1563-1570.

Holzhey DM, Jacobs S, Mochalski M, Merk D, Walther T, Mohr FW, Falk V (2008). Minimally invasive hybrid coronary artery revascularization. Ann. Thorac. Surg. 86(6):1856-60.

Kapetanakis El, Stamou SC, Petro KR, Hill PC, Boyce SW, Bafi AS, Corso PJ (2008). Comparison of the quality of life after conventional versus off-pump coronary artery bypass surgery. J. Card Surg. 23(2):120-125.

Kiernan PD, Khandhar SJ, Fortes DL, Schmidt K, Sheridan MJ, Hetrick V (2011). Thoracic Surgery in Octogenarians CVTSA/Inova Fairfax 
hospital experience, 1990 to 2009. Am. Surg. 77(6):675-80.

Kugler C, Altenhöner T, Lochner P, Ferbert A (2003). Hessian Stroke Data Bank Study Group ASH: Does age influence early recovery from ischemic stroke? A study from the Hessian Stroke Data Bank. J. Neurol. 250(6):676-81.

Kuss O, von Salviati B, Borgermann J (2010). Off-pump versus onpump coronary artery bypass grafting: a systematic review and metaanalysis of propensity score analyses. J. Thorac. Cardiovasc. Surg. 140(4):829-35, 835.

Lim E, Drain A, Davies W, Edmonds L, Rosengard BR (2006). A systematic review of randomized trials comparing revascularization rate and graft patency of off-pump and conventional coronary surgery. J. Thorac. Cardiovasc. Surg. 132(6):1409-13.

Mishra M, Malhotra R, Karlekar A, Mishra Y, Trehan N (2006). Propensity case-matched analysis of off-pump versus on-pump coronary artery bypass grafting in patients with atheromatous aorta. Ann. Thorac. Surg. 82(2):608-14.

Motallebzadeh R, Bland JM, Markus HS, Kaski JC, Jahangiri M (2006). Health-related quality of life outcome after on-pump versus off-pump coronary artery bypass graft surgery: a prospective randomized study. Ann. Thorac. Surg. 82(2):615-9.

OECD StatExtracts. Demographic References. Population age structure. Available

http://stats.oecd.org/index.aspx?DataSetCode=HEALTH_STAT Accessed August 19, 2011.

Organization for Economic Co-operation and Development (OECD). Health at a Glance OECD indicators (2005) OECD Publishing, Paris, p56.
Rivera C, Dahan M, Bernard A, Falcoz PE, Thomas P (2011). Surgical treatment of lung cancer in the octogenarians: results of a nationwide audit. Eur. J. Cardiothorac. Surg. 39(6):981-6.

Sacanella E, Pérez-Castejón JM, Nicolás JM, Masanés F, Navarro M, Castro P, López-Soto A (2011). Functional status and quality of life 12 months after discharge from a medical ICU in healthy elderly patients: a prospective observational study. Crit. Care. 15(2): R105.

Suaya JA, Stason WB, Ades PA, Normand SL, Shepard DS (2009). Cardiac rehabilitation and survival in older coronary patients. J. Am. Coll. Cardiol. 54(1):25-33.

Sulter G, Steen C, Keyser J (1999) Use of the Barthel Index and Modified Rankin Scale in acute stroke trials. Stroke 30(8):1538-41.

Sundararajan V, Henderson T, Perry C, Muggivan A, Quan H, Ghali WA (2004). New ICD-10 version of the Charlson Comorbidity Index predicted in-hospital mortality. J. Clin. Epidemiol. 57(12):1288-94.

The Ministry of Health, Labour and Welfare. Available at http://www.mhlw.go.jp/stf/shingi/2r985200000196c4att/2r985200000196gc.pdf. Accessed 21 August, 2011.

Zhan C, Miller MR (2003). Administrative data based patient safety research: a critical review. Qual. Saf. Health Care 12(Suppl 2): ii58ii63. 
Annex Table 1. Comparison of patient characteristics, care process and outcomes between the excluded and included population (\%) [SD].

\begin{tabular}{|c|c|c|c|c|c|c|}
\hline \multirow{2}{*}{ Parameter } & \multicolumn{3}{|c|}{ CABG } & \multicolumn{3}{|c|}{ Lung lobectomy } \\
\hline & Excluded group & Included group & $\mathbf{P}$ & Excluded group & Included group & $\mathbf{P}$ \\
\hline Patient number (academic, community) & 13523 & 7573 & & 33490 & 20407 & \\
\hline Hospital number (academic, community) & 78,302 & 36,131 & & 81,609 & 37,218 & \\
\hline \multicolumn{7}{|l|}{ Innovation } \\
\hline Off pump or VAL & $7093(52.5)$ & $3672(48.5)$ & & $22168(66.2)$ & $14378(70.5)$ & $<0.001$ \\
\hline Mean, [SD] & $68.2[9.5]$ & $68.3[9.5]$ & $0.329^{\dagger}$ & $66.7[11.4]$ & $67.2[10.6]$ & $<0.001^{\dagger}$ \\
\hline \multicolumn{7}{|l|}{ Age } \\
\hline Under 15 years & $7(0.1)$ & $0(0.0)$ & \multirow{4}{*}{0.188} & $186(0.6)$ & $0(0.0)$ & \multirow{4}{*}{$<0.001$} \\
\hline $15-64$ years & $4365(32.3)$ & $2410(31.8)$ & & $12052(36.0)$ & $7267(35.6)$ & \\
\hline $65-74$ years & $5399(39.9)$ & 3019 (39.9) & & $12560(37.5)$ & 7661 (37.5) & \\
\hline$\geq 75$ years & $3752(27.7)$ & $2144(28.3)$ & & $8692(26.0)$ & $5479(26.8)$ & \\
\hline \multicolumn{7}{|l|}{ Gender } \\
\hline Male & $10299(76.2)$ & $5791(76.5)$ & 0.612 & $20555(61.4)$ & $12669(62.1)$ & 0.102 \\
\hline \multicolumn{7}{|l|}{ Ambulance } \\
\hline Used & $2084(15.4)$ & $951(12.6)$ & $<0.001$ & $124(0.4)$ & $54(0.3)$ & 0.038 \\
\hline \multicolumn{7}{|l|}{ Charlson comorbidity index } \\
\hline 1 & 4695 (34.7) & $2732(36.1)$ & \multirow{4}{*}{0.164} & $6308(18.8)$ & $4139(20.3)$ & \multirow{4}{*}{$<0.001$} \\
\hline 2 & $3035(22.4)$ & $1613(21.3)$ & & $5192(15.5)$ & $3264(16.0)$ & \\
\hline 3 & $1080(8.0)$ & $628(8.3)$ & & $2156(6.4)$ & $1433(7.0)$ & \\
\hline 4 or more & $476(3.5)$ & $270(3.6)$ & & $1315(3.9)$ & $787(3.9)$ & \\
\hline \multicolumn{7}{|l|}{ Teaching status } \\
\hline Academic & $4241(31.4)$ & $1818(24)$ & $<0.001$ & $10411(31.1)$ & $6734(33.0)$ & $<0.001$ \\
\hline \multicolumn{7}{|l|}{ Fiscal year } \\
\hline 2006 & $1537(44.4)$ & $1925(55.6)$ & & $2890(40.6)$ & $4233(59.4)$ & \\
\hline 2007 & $3517(73.6)$ & $1260(26.4)$ & & $8472(74.6)$ & $2889(25.4)$ & \\
\hline 2008 & $3496(74.7)$ & $1183(25.3)$ & $<0.001$ & $8140(73.0)$ & $3004(27.0)$ & $<0.001$ \\
\hline 2009 & $2111(60.9)$ & 1357 (39.1) & & $5685(58.7)$ & 3996 (41.3) & \\
\hline 2010 & $2862(60.8)$ & 1848 (39.2) & & 8303 (56.9) & $6285(43.1)$ & \\
\hline \multicolumn{7}{|l|}{$\mathrm{PCl}$} \\
\hline Overall & $929(6.9)$ & $491(6.5)$ & 0.283 & & $* * *$ & \\
\hline Preoperative & 499 (3.7) & $243(3.2)$ & 0.069 & & & \\
\hline Postoperative & $463(3.4)$ & $257(3.4)$ & 0.908 & & & \\
\hline
\end{tabular}


Annex Table 1. Contd.

\begin{tabular}{|c|c|c|c|c|c|c|}
\hline \multirow{2}{*}{ Parameter } & \multicolumn{3}{|c|}{ CABG } & \multicolumn{3}{|c|}{ Lung lobectomy } \\
\hline & Excluded group & Included group & $\mathbf{P}$ & Excluded group & Included group & $\mathbf{P}$ \\
\hline \multicolumn{7}{|l|}{ ICU stay } \\
\hline Overall & $11538(85.3)$ & $6364(84)$ & 0.012 & $11788(35.2)$ & $6476(31.7)$ & $<0.001$ \\
\hline Preoperative & $1975(14.6)$ & $1037(13.7)$ & 0.07 & $75(0.2)$ & $32(0.2)$ & 0.089 \\
\hline Postoperative & $11373(84.1)$ & $6295(83.1)$ & 0.065 & $11756(35.1)$ & $6468(31.7)$ & $<0.001$ \\
\hline \multicolumn{7}{|l|}{ Blood transfusion } \\
\hline Overall & $8664(64.1)$ & $4893(64.6)$ & 0.430 & $2001(6)$ & $1171(5.7)$ & 0.257 \\
\hline Preoperative & $468(3.5)$ & $207(2.7)$ & 0.004 & $151(0.5)$ & $75(0.4)$ & 0.146 \\
\hline Postoperative & $8634(63.8)$ & $4881(64.5)$ & 0.379 & $1922(5.7)$ & $1138(5.6)$ & 0.429 \\
\hline \multicolumn{7}{|l|}{ Ventilation } \\
\hline Overall & $9281(68.6)$ & $4938(65.2)$ & $<0.001$ & $834(2.5)$ & $443(2.2)$ & 0.018 \\
\hline Preoperative & $382(2.8)$ & $176(2.3)$ & 0.030 & $41(0.1)$ & $10(0.0)$ & 0.007 \\
\hline Postoperative & $9227(68.2)$ & $4904(64.8)$ & $<0.001$ & $813(2.4)$ & $438(2.1)$ & 0.035 \\
\hline \multicolumn{7}{|l|}{ Cardiac support device } \\
\hline Overall & $2740(20.3)$ & $1377(18.2)$ & $<0.001$ & & & \\
\hline Preoperative & $949(7.0)$ & $416(5.5)$ & $<0.001$ & & $* * *$ & \\
\hline Postoperative & $2508(18.5)$ & $1285(17.0)$ & 0.004 & & & \\
\hline \multicolumn{7}{|l|}{ Blood purification } \\
\hline Overall & $1467(10.8)$ & 749 (9.9) & 0.030 & $241(0.7)$ & $161(0.8)$ & 0.364 \\
\hline Preoperative & $1027(7.6)$ & $547(7.2)$ & 0.325 & $212(0.6)$ & $136(0.7)$ & 0.639 \\
\hline Postoperative & $1428(10.6)$ & $731(9.7)$ & 0.037 & $238(0.7)$ & $158(0.8)$ & 0.402 \\
\hline \multicolumn{7}{|l|}{ Chemoradiation } \\
\hline Chemotherapy & & & & $1426(4.3)$ & $685(3.4)$ & \\
\hline Radiation & & $* * *$ & & $80(0.2)$ & $39(0.2)$ & $<0.001$ \\
\hline Chemoradiation & & & & $91(0.3)$ & $39(0.2)$ & \\
\hline Operating room time (minute) & $435.7[273.4]$ & $443.3[164.4]$ & $0.029^{\dagger}$ & $285.3[112.5]$ & $294.6[206]$ & $0.772^{\dagger}$ \\
\hline \multicolumn{7}{|l|}{ Complication } \\
\hline Minor complication & $2423(17.9)$ & $1496(19.8)$ & 0.004 & $4378(13.1)$ & $2356(11.5)$ & $<0.001$ \\
\hline Major complication & $49(0.4)$ & $27(0.4)$ & - & $92(0.3)$ & $52(0.3)$ & - \\
\hline Abscess drainage & $17(0.1)$ & $8(0.1)$ & 0.684 & $45(0.1)$ & $24(0.1)$ & 0.598 \\
\hline Operative hemostasis & $32(0.2)$ & $20(0.3)$ & 0.700 & $50(0.1)$ & $30(0.1)$ & 0.947 \\
\hline
\end{tabular}

CABG: coronary artery bypass graft, VAL: video assisted lobectomy, SD: standard deviation, $\uparrow$ : compared using analysis of variance. Other comparisons were made using chi-square test. $\mathrm{BI}$ : barthel index, ICU: intensive care unit, $\mathrm{PCl}$ : percutaneous coronary intervention, ${ }^{* \star *}$ : not examine. 